Submitted to the Annals of Applied Statistics

arXiv: math.PR/0000000

\title{
SUPPLEMENTARY MATERIAL: MODELING CONCURRENCY AND SELECTIVE MIXING IN HETEROSEXUAL PARTNERSHIP NETWORKS WITH APPLICATIONS TO SEXUALLY TRANSMITTED DISEASES
}

\author{
By Ryan Admiraal and Mark S. Handcock
}

Figure S1 is a barplot of the HIV prevalence for sub-Saharan African countries based on their Demographic and Health Surveys (DHS) estimates [Demographic and Health Surveys Program, 2015].

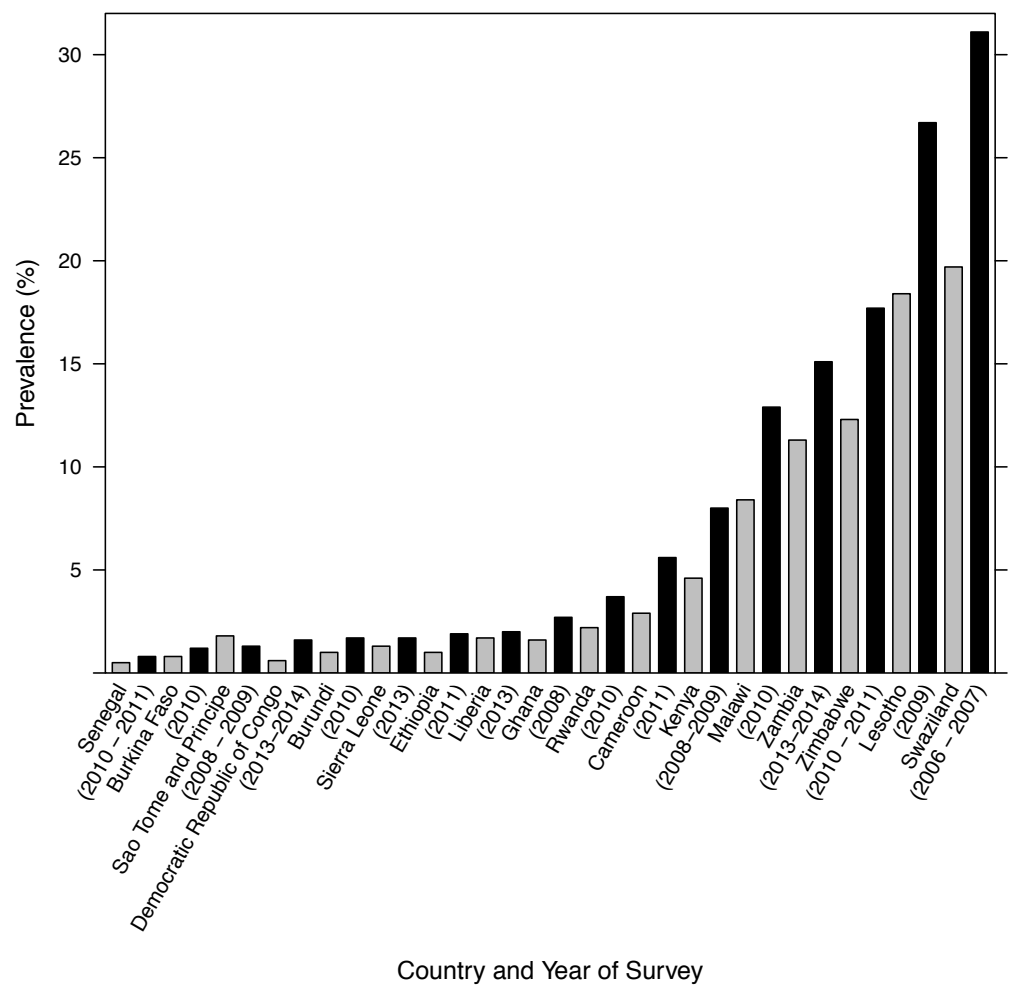

Fig S1: HIV prevalence for males (gray) and females (black) ages 15-49 years in select sub-Saharan Africa countries. Source: Demographic and Health Surveys Program [2015]. 
S1. The Measurement of Concurrency. Historically, concurrency has been measured "directly" through individuals' self-reports of multiple partnerships being current and sexual or "indirectly" through individuals' reported first and last sexual encounters with each partner over a prescribed period of time [Helleringer et al., 2014]. Even within these two approaches there has been variability in how concurrency has been measured, and, in an attempt to produce consistency in the way that concurrency is understood and measured, the UNAIDS Reference Group on Estimates, Modelling, and Projections [2009] defined concurrency as "Overlapping sexual partnerships where sexual intercourse with one partner occurs between two acts of intercourse with another partner." It produced a series of recommendations in terms of how concurrency should be measured, including specifying that the point prevalence of concurrency be the preferred measure of concurrency. It also supported two additional point estimators for concurrency, and the descriptions of these three estimators are presented in Table S1.

\begin{tabular}{ll} 
Estimator & Description \\
\hline $\begin{array}{l}\text { Point prevalence of con- } \\
\text { current partnerships }\end{array}$ & $\begin{array}{l}\text { Percentage of the population with concurrent partnerships } \\
\text { at a given point in time, specified to be six months prior to } \\
\text { the interview according to the UNAIDS Reference Group } \\
\text { recommendations. }\end{array}$ \\
$\begin{array}{l}\text { Cumulative prevalence of } \\
\text { concurrent partnerships }\end{array}$ & $\begin{array}{l}\text { Percentage of the population with concurrent partnerships } \\
\text { over a given period of time, specified to be the year pre- } \\
\text { ceding the interview according to the UNAIDS Reference } \\
\text { Group recommendations. }\end{array}$ \\
$\begin{array}{l}\text { Proportion of multiple } \\
\text { partnerships which are } \\
\text { concurrent }\end{array}$ & $\begin{array}{l}\text { Percentage of the population reporting multiple partner- } \\
\text { ships (which may be concurrent or may be "sequentially } \\
\text { monogamous") that also reported concurrent partnerships } \\
\text { over a given period of time, specified to be the year pre- } \\
\text { ceding the interview according to the UNAIDS Reference } \\
\text { Group recommendations. }\end{array}$ \\
\hline
\end{tabular}

TABLE S1

Point estimators for concurrency recommended by the UNAIDS Reference Group on Estimates, Modeling, and Projections.

The point estimators for concurrency recommended by the UNAIDS Reference Group are all based on the indirect approach of measuring concurrency with the preferred estimator being a retrospective measure at six months prior to the time of the interview. Although the direct approach would be expected to be less susceptible to recall bias because it is based on partnerships at the time of the interview, Helleringer et al. [2014] note that the direct approach is speculative and, consequently, may be biased. This is 
because a respondent cannot know for certain whether or not partnerships deemed to be current or ongoing at the time of the interview will produce an overlap in sexual encounters corresponding to that time point, as this requires a future sexual encounter involving one of these partners.

Using sexual partnership data collected in KwaZulu-Natal, South Africa, from surveys following the UNAIDS Reference Group recommendations, Eaton et al. [2012] suggest that the direct approach appears to produce positively biased estimates for concurrency for this region with this bias being attributable almost completely to men under the age of 30 . They examined the point prevalence of concurrency based on a range of retrospective time points and found that point prevalence for retrospective time points of 4-6 months was fairly consistent and higher than other time points, supporting the UNAIDS Reference Group recommended retrospective period of 6 months. In the case of more recent time points, lower estimates may in part be explained by either long-term partnerships where one partnership has not produced a sexual encounter or by recent partnerships that a respondent may be reluctant to reveal. For more distant time points, recall bias may lead to missing data for sexual encounters or failure to report partnerships.

In contrast to the findings of Eaton et al. [2012] for South Africa, Goodreau et al. [2012] found that point prevalence of concurrency was highest at 2 months prior to interview for data from Zimbabwe [NIMH Collaborative HIV/STD Prevention Trial Group, 2007]. This would suggest that choice of an appropriate retrospective period for the indirect approach is not necessarily clear and may depend on the anticipated level of recall bias and frequency of sexual contact for those engaged in concurrent partnerships. This lends credence to the notion of concurrency being difficult to precisely define or measure "because it represents a spectrum of different types of sexual relationships with varying durations and overlaps" [Lurie and Rosenthal, 2010], so, even if the proposed form of point estimator is clear, the best time point at which to measure it is not.

In an attempt to take into consideration partnership duration and length of overlap of concurrent partnerships, Lagarde et al. [2001] proposed the "individual indicator of concurrency" (iic) which measures the duration of overlap in concurrent partnerships and what would be expected based on the length of these relationships. This produces an individual-specific measure which can be aggregated to produce a population-level estimate. While the durations of not only individual partnerships but also overlaps in concurrent partnerships have ramifications for disease transmission, these are not measures of concurrency in the strict sense but rather can be consid- 
ered as separate vital considerations for the disease transmission process. (Both partnership durations and duration of overlap of concurrent partnerships can be used to inform the parameters governing partnership formation and dissolution in network-based simulations, but they do not influence the structure of an initial network, which is the focus of this paper.) The same could be said for coital frequency (Gaydosh et al. [2013] find some evidence for coital dilution [i.e. less frequent coital events] for those engaged in concurrent partnerships) and transmission rates per coital event (which may change depending on the stage of infection for HIV-positive individuals [Hollingsworth et al., 2008]; coinfections of other STDs [Fleming and Wasserheit, 1999], schistosomiasis [Kjetland et al., 2012], and malaria [AbuRaddad et al., 2006]; whether a male is circumcised [Szabo and Short, 2010]; level of condom use [Davis and Weller, 1999]; etc.).

Although the point estimators recommended by the UNAIDS Reference Group provide a clear measure of concurrency according to the Group's proposed definition, they sacrifice additional information that is relevant to disease transmission. In particular, they provide no information about just how many partners those with concurrent partnerships tend to have. Kretzschmar and Morris [1996] propose point estimators that are meant to incorporate this information in attempting to measure concurrency for a network representing individuals (as nodes) and partnerships (as edges). They first present estimators based on the degree mean (i.e. average number of partnerships per person) for the original network and network density (i.e. proportion of actual partnerships out of total possible partnerships) for a line graph constructed by letting edges (i.e. partnerships) in the original network represent nodes and adjacent edges (i.e. concurrent partnerships) represent an edge between nodes. These are ultimately deemed to be deficient due to failure to take into consideration the connectedness of the original network in the case of the first estimator and the percentage of isolates (i.e. individuals with no partners) in the original network in the case of the second estimator. A third estimator of network-level concurrency, referred to as the $\kappa$-statistic, was proposed which was also based on the line graph of ties but could be shown to be a function of the mean and variance of the degree distribution for the original network. It represents the average number of concurrent partnerships per partnership in the population of interest. This proves beneficial in that it does not require full network data for estimation, so local network (including cross-sectional) data can be used for estimation of concurrency.

More recent modeling approaches have moved away from point estimators of concurrency and instead incorporate full momentary degree distributions 
(from now on simply referred to as "degree distributions"), the distribution of numbers of sexual partnerships for individuals at a given point in time (e.g. Morris et al. [2009], Hamilton et al. [2008], Carnegie and Morris [2011], Goodreau et al. [2012]). Degree distributions preserve more of the epidemiologically relevant information than a single point estimator while being able to recover both the $\kappa$-statistic and point prevalence of concurrency. In particular, the proportion of the sexually active population with a momentary degree greater than 1 exactly matches the point prevalence of concurrency. At the same time, since the $\kappa$-statistic is a function of the mean and variance of the degree distribution, the degree distribution can easily recover the $\kappa$-statistic. Consequently, we use degree distributions rather than more commonly reported point estimators of concurrency.

Like point estimators for concurrency, degree distributions are flexible and can be stratified according to a variety of characteristics that may be associated with prevalence of concurrency. For example, Wellings et al. [2006] find higher levels of concurrency for men than women based on data from 59 countries including the United States, Australia, China, Chile, and much of Africa and western Europe. Using data from the 2002 National Survey of Family Growth, Adimora et al. [2007] find that concurrency is related to race/ethnicity, and using data from the National Longitudinal Study of Adolescent Health [Harris et al., 2009], Hamilton and Morris [2015] show that one-year cumulative prevalence for non-Hispanic black Americans is roughly double that of non-Hispanic white Americans. Based on data from South Africa, Eaton et al. [2011] show evidence for higher concurrency for males aged 20-34 years than other age groups. And, in an examination of data collected in Zambia, Sandøy et al. [2010] found that engagement in concurrent partnerships was related to age at first sexual encounter, marital status, age at marriage, and absence from the home. Stratifying for sex, age, race, and other factors related to differential rates of concurrency can help ensure that initial networks for disease modeling more accurately reflect partnership networks in the population of interest.

\section{S2. The Relationship Between Concurrency and Disease Spread.}

S2.1. Arguments for the relevance of concurrency to disease spread. A number of studies have claimed that concurrency is important in explaining disparities in STI prevalence and the speed with which STIs propagate through a population [Watts and May, 1992, Hudson, 1993, Morris and Kretzschmar, 1995, 1997, 2000, Kretzschmar and Morris, 1996, Ghani et al., 1997, Chick et al., 2000, Ghani and Garnett, 2000, Koopman et al., 2000, Adimora and Schoenbach, 2002, 2005, Adimora et al., 2006, 2007, Do-

imsart-aoas ver. 2012/08/31 file: Supplement2.tex date: June 4, 2016 
herty et al., 2006, Morris et al., 2007, 2009, Johnson et al., 2009, Mah and Halperin, 2010, Eaton et al., 2011, Goodreau, 2011, Goodreau et al., 2012, Kretzschmar and Caraël, 2012, Hamilton and Morris, 2015]. If we consider a population under serial monogamy, current relationships must end before infected individuals place other non-infected individuals at risk. With concurrency, on the other hand, if an individual has concurrent sexual partners and becomes infected by one, the other partners are placed at risk almost immediately. Consequently, greater connectivity of a population that is possible under higher levels of concurrency should theoretically provide a much more efficient mechanism for disease spread.

In a series of simulation studies, Morris and Kretzschmar [1995, 1997] and Kretzschmar and Morris [1996] investigated the effects of concurrent partnerships on the spread of STD epidemics and found that concurrency led to much more rapid spread of the epidemic in its initial phase, and the expected number of infected individuals was approximately an exponential function of the level of concurrency (as measured by the $\kappa$-statistic). A later simulation study using observed levels of concurrency based on data from Uganda concluded that, under proportional mixing (i.e. random partner selection) and when compared to serial monogamy, the observed level of concurrency led to an expected $26 \%$ increase in the number of infected individuals in the population after five years [Morris and Kretzschmar, 2000].

In their examination of HIV prevalence in the African-American population in the United States, Adimora and Schoenbach [2002, 2005] and Adimora et al. [2006] similarly concluded that concurrency is an important factor in the disparities observed between blacks and other races. Similarly, simulations by Morris et al. [2009] showed that, based on observed levels of concurrency and race-based selective mixing from the National Longitudinal Study of Adolescent Health [Harris et al., 2009], concurrency increased the predicted disparity in HIV prevalence between non-Hispanic black and white Americans by a multiplicative factor of 2.6. They found that even slight changes to the level of concurrency led to dramatic changes in this predicted disparity, so even small changes in the level of concurrency could have a significant impact on the spread of the disease.

More recent simulations by Eaton et al. [2011] that take into consideration stage-specific HIV transmission rates based on data from Uganda find that HIV prevalence grows with the level of concurrency (as estimated by both point prevalence of concurrency and the $\kappa$-statistic), and "Indeed, with staged transmission, concurrency makes the difference as to whether an epidemic can spread at all." Goodreau et al. [2012] extend the Eaton et al. [2011] model, allowing for differential concurrency by gender and number of 
cohabiting and non-cohabiting partners.

In these studies, selective mixing is not incorporated for the populations considered (meaning that random mixing is assumed), and simulations assume populations of equal numbers of men and women. (The lone exception to this is Morris and Kretzschmar [1995], who incorporate mixing but do so only for symmetric levels of assortativity [or preferences for partnerships with those of similar characteristics], meaning that propensities for partnerships within subpopulations are the same for all subpopulations, and propensities for partnerships between subpopulations are constrained to be equal.) In the case of differential concurrency by gender and other factors as considered by Goodreau et al. [2012] these were handled in an ad hoc manner, averaging the mean number of partners reported by men and women, respectively, and recoding partnership information for several individuals to ensure consistency between male and female reports of total number of partnerships.

S2.2. Critiques of the concurrency hypothesis. In contrast to previously cited studies suggesting the importance of concurrency in explaining higher rates of HIV prevalence in sub-Saharan Africa and for non-Hispanic blacks in the United States, a number of studies refute the concurrency hypothesis. Lurie and Rosenthal [2010], Sawers and Stillwagon [2010], and Sawers [2013] present overviews of empirical evidence for the relationship between concurrency and HIV risk, noting discrepancies in how concurrency is measured across multiple studies, thereby compromising the validity of many comparisons used to claim that concurrency is important in explaining HIV risk. They also present results from studies that fail to find evidence supporting the concurrency hypothesis. For example, Lagarde et al. [2001] examined data from urban communities in five sub-Saharan African countries. Using both the $\kappa$-statistic of Kretzschmar and Morris [1996] and the iic, they failed to find significantly higher HIV prevalence for those communities reporting higher levels of concurrency. Similarly, Tanser et al. [2011] found an association between number of sexual partners and HIV risk but not between concurrency and HIV risk using data from KwaZulu-Natal. And Mishra and Assche [2009] claimed that prevalence of HIV does not seem to be associated with prevalence of concurrency for community- or national-level models incorporating DHS and AIDS Indicator Survey (AIS) data from sub-Saharan Africa.

Sawers and Stillwagon [2010] and Sawers [2013] also review the modelbased approaches used to support the importance of concurrency in disease transmission. They highlight common errors in modeling approaches as well 
as specific issues with the assumptions underlying some of the more common modeling methods, including those originally proposed by Morris and Kretzschmar. The errors they mention are wide-ranging, including some modelers mistakenly treating cumulative prevalence estimates as point prevalence estimates of concurrency, the use of unrealistic partnership durations and coital frequency, and transmission rates that are not data-based or that fail to take into account the stage of HIV infection.

Relevant to the production of an initial network, Sawers [2013] notes that a number of studies calculate point prevalence of concurrency using as the denominator the entire population size rather than the population of sexually active or experienced individuals. Additionally, the majority of studies considering heterosexual partnership networks use a single measure of concurrency for men and women together rather than separately for the two sexes, and nearly all use populations with equal numbers of men and women for simulations. We additionally note that, while current modeling approaches commonly incorporate either momentary degree distributions (or other measures of concurrency) or selective mixing totals, few account for the two simultaneously due to the complex relationship between them. Those that do incorporate both have required simplifying assumptions about the nature of selective mixing or concurrency.

S3. Parameterizations of Dependence Parameters for Mixing. The parameterization provided in equation (4) of the paper provides flexibility to consider a variety of dependence structures. For instance, if we want to model uniform homophily (i.e. symmetric assortative mixing), we set

$$
\alpha_{i j}= \begin{cases}\alpha, & i=j \\ \gamma, & i \neq j\end{cases}
$$

for constants $\alpha$ and $\gamma$, and estimated propensities for partnerships with a person of the same type will be identical across all types. We might also consider modeling differential homophily (i.e. asymmetric assortative mixing), in which case same-type propensities need not be the same across types. For differential homophily, we set

$$
\alpha_{i j}=\left\{\begin{array}{cc}
\alpha_{i}, & i=j \\
\gamma, & i \neq j
\end{array}\right.
$$

S4. Maximum Likelihood Estimators for Degree Distribution and Mixing Parameters. To jointly estimate the degree distributions $\pi^{\mathcal{M}}=\left\{\pi_{1}^{\mathcal{M}}, \ldots, \pi_{I}^{\mathcal{M}}\right\}$ and $\pi^{\mathcal{F}}=\left\{\pi_{1}^{\mathcal{F}}, \ldots, \pi_{J}^{\mathcal{F}}\right\}$ and mixing totals $\mu=$ 
$\left(\mu_{i j}\right)_{1 \leq i \leq I, 1 \leq j \leq J}$ described in $\S 3$ of the paper, we use the joint likelihood of $\pi^{\mathcal{M}}, \pi^{\mathcal{F}}$, and $\alpha=\left(\alpha_{i j}\right)_{1 \leq i \leq I, 1 \leq j \leq J}$ given by

$$
\begin{aligned}
& \mathrm{L}\left(\pi^{\mathcal{M}}, \pi^{\mathcal{F}}, \alpha\right)=\left[\prod_{i=1}^{I} \prod_{j=1}^{J} \frac{\exp \left(-\frac{2\left(\sum_{k=0}^{F} k \pi_{i k}^{\mathcal{M}} m_{i}\right)\left(\sum_{\ell=0}^{M} \ell \pi_{j \ell}^{\mathcal{F}} F_{j}\right)}{\left(\sum_{i=1}^{I} \sum_{k=0}^{F} k \pi_{i k}^{\mathcal{M}} M_{i}\right)+\left(\sum_{j=1}^{J} \sum_{\ell=0}^{M} \ell \pi_{j \ell}^{\mathcal{F}} F_{j}\right)} \alpha_{i j}\right)}{n_{i j}^{\mathcal{M} !}}\right. \\
& \cdot\left(\frac{2\left(\sum_{k=0}^{F} k \pi_{i k}^{\mathcal{M}} m_{i}\right)\left(\sum_{\ell=0}^{M} \ell \pi_{j \ell}^{\mathcal{F}} F_{j}\right)}{\left(\sum_{i=1}^{I} \sum_{k=0}^{F} k \pi_{i k}^{\mathcal{M}} M_{i}\right)+\left(\sum_{j=1}^{J} \sum_{\ell=0}^{M} \ell \pi_{j \ell}^{\mathcal{F}} F_{j}\right)} \alpha_{i j}\right)^{n_{i j}^{\mathcal{M}}} \\
& \cdot \frac{\exp \left(-\frac{2\left(\sum_{k=0}^{F} k \pi_{i k}^{\mathcal{M}} M_{i}\right)\left(\sum_{\ell=0}^{M} \ell \pi_{j \ell}^{\mathcal{F}} f_{j}\right)}{\left(\sum_{i=1}^{I} \sum_{k=0}^{F} k \pi_{i k}^{\mathcal{M}} M_{i}\right)+\left(\sum_{j=1}^{J} \sum_{\ell=0}^{M} \ell \pi_{j \ell}^{\mathcal{F}} F_{j}\right)} \alpha_{i j}\right)}{n_{j i}^{\mathcal{F}} !} \\
& \left.\cdot\left(\frac{2\left(\sum_{k=0}^{F} k \pi_{i k}^{\mathcal{M}} M_{i}\right)\left(\sum_{\ell=0}^{M} \ell \pi_{j \ell}^{\mathcal{F}} f_{j}\right)}{\left(\sum_{i=1}^{I} \sum_{k=0}^{F} k \pi_{i k}^{\mathcal{M}} M_{i}\right)+\left(\sum_{j=1}^{J} \sum_{\ell=0}^{M} \ell \pi_{j \ell}^{\mathcal{F}} F_{j}\right)} \alpha_{i j}\right)^{n_{i j}^{\mathcal{F}}}\right] \\
& \cdot\left[\prod_{i=1}^{I}\left(\begin{array}{c}
m_{i} \\
d_{i 0}^{\mathcal{M}}, \cdots, d_{i F}^{\mathcal{M}}
\end{array}\right)\left(\pi_{i 0}^{\mathcal{M}}\right)^{d_{i 0}^{\mathcal{M}}} \cdots\left(\pi_{i F}^{\mathcal{M}}\right)^{d_{i F}^{\mathcal{M}}}\right. \\
& \left.\cdot \prod_{j=1}^{J}\left(\begin{array}{c}
f_{j}^{\mathcal{F}} \\
d_{j 0}^{\mathcal{F}}, \cdots, d_{j M}^{\mathcal{F}}
\end{array}\right)\left(\pi_{j 0}^{\mathcal{F}}\right)^{d_{j 0}^{\mathcal{F}}} \cdots\left(\pi_{j M}^{\mathcal{F}}\right)^{d_{j M}^{\mathcal{F}}}\right] .
\end{aligned}
$$

S5. Estimating Natural Parameters from Mean Value Parameters. To simulate networks consistent with desired mixing totals and degree distributions, we treat the estimated expected degree distributions and mixing totals as mean value parameters for an exponential-family random graph model (ERGM). ERGMs are a class of probabilistic models for networks that take on the classic exponential-family form. Holland and Leinhardt [1981] considered an instance of this class of models when they proposed the $p_{1}$ model, a model that assumes independence between dyads. Later developments by Frank and Strauss [1986], Strauss and Ikeda [1990], and Wasserman and Pattison [1996] generalized this model to produce the $p^{*}$ model, the standard ERGM. This model takes on the form

$$
P(Y=y \mid \theta)=\frac{\exp (\theta \cdot g(y))}{\kappa(\theta)},
$$

where $y$ is a realization of some random network $Y, g(y)$ is a vector of sufficient statistics, $\theta$ is the vector of corresponding natural parameters, and 
$\kappa(\theta)$ is the normalizing constant, given by

$$
\kappa(\theta)=\sum_{x \in \mathcal{Y}} \exp (\theta \cdot g(x))
$$

where $\mathcal{Y}$ is the network space. Hunter and Handcock [2006] show that inference can be carried out on an observed network for both standard and curved ERGMs (ERGMs where $\theta$ can be expressed as a non-linear function of some lower dimension parameter) using Markov chain Monte Carlo (MCMC) maximum likelihood estimation [Geyer and Thompson, 1992].

In addition to its use in maximum likelihood estimation for ERGMs, MCMC has the added benefit of providing a mechanism for the simulation of new networks consistent with a vector of natural parameters $\theta$. Hunter et al. [2008] describe how this can be done using Gibbs sampling, a Metropolis algorithm, or a Metropolis-Hastings algorithm. This method is fairly robust, allowing for the introduction of constraints on a number of network statistics [Morris et al., 2008].

The expected degree distributions $\pi^{\mathcal{M}}$ and $\pi^{\mathcal{F}}$ and expected mixing totals $\mu$ provide population-level estimates, but they cannot be directly used by an ERGM to simulate networks consistent with these structures. However, they can be used to estimate the natural parameters $\theta$ for an ERGM, thereby enabling us to simulate the desired networks. To demonstrate how this is done, suppose we want to estimate the natural parameters $\theta$ for a vector of sufficient statistics $g(y)$. Handcock [2003] represents the mean value parameterization as

$$
\mu(\theta)=\mathbb{E}_{\theta}[g(Y)]
$$

the expected value over the parameter space of the sufficient statistics.

If we recall the form of the ERGM given by (S2) and corresponding normalizing constant given by (S3), then the logarithm of the ERGM is given by

$$
\log P(Y=y \mid \theta)=\theta \cdot g(y)-\psi(\theta)
$$

where

$$
\psi(\theta)=\log \left[\sum_{x \in \mathcal{Y}} \exp (\theta \cdot g(x))\right]
$$

imsart-aoas ver. 2012/08/31 file: Supplement2.tex date: June 4, 2016 
Then the mean value parameters (S4) can be expressed as

$$
\begin{aligned}
\mu(\theta) & =\sum_{y \in \mathcal{Y}} g(y) P(Y=y \mid \theta) \\
& =\sum_{y \in \mathcal{Y}} g(y) \frac{\exp (\theta \cdot g(y))}{\sum_{x \in \mathcal{Y}} \exp (\theta \cdot g(x))} \\
& =\frac{\partial \psi(\theta)}{\partial \theta},
\end{aligned}
$$

and the covariance matrix corresponding to these mean value parameters is given by

$$
\begin{aligned}
\Sigma(\theta) & =\sum_{y \in \mathcal{Y}} g(y)^{2} P(Y=y \mid \theta)-\mu(\theta)^{T} \mu(\theta) \\
& =\sum_{y \in \mathcal{Y}} g(y) \frac{\exp (\theta \cdot g(y))}{\sum_{x \in \mathcal{Y}} \exp (\theta \cdot g(x))} g(y)^{T}-\left[\sum_{y \in \mathcal{Y}} g(y) \frac{\exp (\theta \cdot g(y))}{\sum_{x \in \mathcal{Y}} \exp (\theta \cdot g(x))}\right]^{2} \\
& =\frac{\partial^{2} \psi(\theta)}{\partial \theta \cdot \partial \theta}
\end{aligned}
$$

In the case where the natural parameters $\theta$ are known and the mean value parameters $\mu(\theta)$ are unknown, it is straightforward to estimate the mean value parameters and their corresponding variances by simulating networks from the network space. If we simulate $L$ new networks $y_{1}, \ldots, y_{L}$ independently and uniformly for a specific value of $\theta$, then we can estimate $\mu(\theta)$ and $\Sigma(\theta)$ by

$$
\hat{\mu}(\theta)=\frac{1}{L} \sum_{i=1}^{L} g\left(y_{i}\right)
$$

and

$$
\hat{\Sigma}(\theta)=\frac{1}{L} \sum_{i=1}^{L} g\left(y_{i}\right) g\left(y_{i}\right)^{T}-\hat{\mu}(\theta)^{T} \hat{\mu}(\theta),
$$

respectively.

Often, the natural parameters are unknown, but desired mean value parameters $\mu_{\text {target }}$ are known, as these correspond to network statistics that can be easily calculated from cross-sectional data. Throughout we consider the case where the natural parameters are finite so that $\mu_{\text {target }}$ is inside the 
convex hull of the sample space of $g(Y)$ [Barndorff-Nielsen, 1978]. Consequently, (S4) takes on the form

$$
\mu_{\text {target }} \equiv \mu\left(\theta_{\text {target }}\right)=\mathbb{E}_{\theta_{\text {target }}}[g(Y)]
$$

and (S5) takes on the form

$$
\mu_{\text {target }}=\sum_{y \in \mathcal{Y}} g(y) \frac{\exp \left(\theta_{\text {target }} \cdot g(y)\right)}{\sum_{x \in \mathcal{Y}} \exp \left(\theta_{\text {target }} \cdot g(x)\right)},
$$

where $\mu_{\text {target }}$, the vector of desired mean value parameters, is known but $\theta_{\text {target }}$, the vector of corresponding natural parameters, is unknown. Solving for $\theta_{\text {target }}(\mu)$ requires that we solve

$$
0=\mu_{\text {target }}-\sum_{y \in \mathcal{Y}} g(y) \frac{\exp \left(\theta_{\text {target }} \cdot g(y)\right)}{\sum_{x \in \mathcal{Y}} \exp \left(\theta_{\text {target }} \cdot g(x)\right)} .
$$

Barndorff-Nielsen [1978] shows that $\theta_{\text {target }}(\mu)$ exists, is the unique solution to (S6), and can be found using a root-finding algorithm, such as NewtonRaphson. Moreover, the Fisher information for $\theta_{\text {target }}$ (i.e. the natural parameterization $)$ is given by $\Sigma\left(\theta_{\text {target }}\right)$, and the Fisher information for the mean value parameter $\mu_{\text {target }}$ is given by $\Sigma^{-1}\left(\theta_{\text {target }}\right)$.

S6. Assessing the Efficacy of the Statistical Approach. To demonstrate the efficacy of our method outlined in $\S 3$ of the paper, we carry out a simulation study based on hypothetical populations with differing levels of concurrency and mixing. For each population, we first demonstrate that the method used for simultaneously estimating degree distributions and mixing totals is able to effectively recapture population degree distributions and mixing totals. Then, using the population degree distribution and mixing totals as mean value parameters, we show that the method of estimating natural parameters for an ERGM from mean value parameters and simulating networks based on those natural parameters is able to generate networks consistent with the mean value parameters. Finally, we demonstrate that additional mean value parameters can be considered, including dyad-dependent statistics such as 3-paths and 4-cycles, and we are able to simulate networks consistent not only with degree distributions and selective mixing totals but also these additional network statistics.

S6.1. Populations for Simulation Studies. Throughout, we consider two hypothetical populations. The first is a population similar to the population of non-Hispanic black and white males and females considered in the

imsart-aoas ver. 2012/08/31 file: Supplement2.tex date: June 4, 2016 
National Longitudinal Survey of Adolescent Health (Add Health), which we refer to as "Faux Add Health." Faux Add Health consists of $20 \%$ nonHispanic blacks and $80 \%$ non-Hispanic whites with equal representation of males and females. Degree distributions are positively skewed, and there is strong assortative mixing by race, in line with what is observed for the Add Health survey. We constrain degree distributions to be the same for males and females as well as both races, and the common degree distribution as well as cell-specific dependence parameters for selective mixing are presented in the top panel of Table S2.

TABLE S2

Degree distributions for non-Hispanic black and white males and females (top left) and cell-specific dependence parameters (top right), along with degree totals (bottom left) and mixing totals (bottom right) based on a population of 2,000 individuals for Faux Add Health.

\begin{tabular}{c|r} 
Degree & Proportion \\
\hline 0 & 0.40 \\
1 & 0.50 \\
2 & 0.06 \\
3 & 0.03 \\
4 & 0.01 \\
\hline
\end{tabular}

\begin{tabular}{cccc} 
& \multicolumn{2}{c}{ Females } \\
& & \multicolumn{1}{c}{ Black } & White \\
\cline { 3 - 4 } Males & Black & $\alpha_{B B}=6$ & $\alpha_{B W}=\frac{3}{8}$ \\
& White & $\alpha_{W B}=\frac{3}{8}$ & $\alpha_{W W}=\frac{57}{32}$ \\
\cline { 3 - 4 } & &
\end{tabular}

\begin{tabular}{c|rrrr} 
Degree & $\begin{array}{c}\text { Black } \\
\text { Males }\end{array}$ & $\begin{array}{c}\text { White } \\
\text { Males }\end{array}$ & $\begin{array}{c}\text { Black } \\
\text { Females }\end{array}$ & $\begin{array}{c}\text { White } \\
\text { Females }\end{array}$ \\
\hline 0 & 80 & 320 & 80 & 320 \\
1 & 100 & 400 & 100 & 400 \\
2 & 12 & 48 & 12 & 48 \\
3 & 6 & 24 & 6 & 24 \\
4 & 2 & 8 & 2 & 8 \\
\hline Pop. Size & 200 & 800 & 200 & 800 \\
\hline
\end{tabular}

\begin{tabular}{ccrr} 
& \multicolumn{3}{c}{ Females } \\
\cline { 3 - 4 } Males & Black & White \\
\cline { 3 - 4 } & Black & 120 & 30 \\
& White & 30 & 570 \\
\cline { 3 - 4 } & &
\end{tabular}

We consider a population of 2,000 individuals where population compositions, degree totals, and mixing totals for Faux Add Health match those presented in the bottom panel of Table S2. We also artificially constrain there to be one hundred and eighty 3-paths and five 4-cycles in the population. Later, we will examine the number of 3-paths and 4-cycles when demonstrating how additional network statistics can be incorporated in our method. A plot of the underlying network of partnerships for Faux Add Health is presented in Figure S2. Note that this plot excludes isolates (i.e. individuals with no partners) in order to better highlight the structure of the network of partnerships.

The second population considered is substantially different from Faux Add Health in terms of racial composition, degree distributions, and selective mixing. This population has equal representation of both races (for 


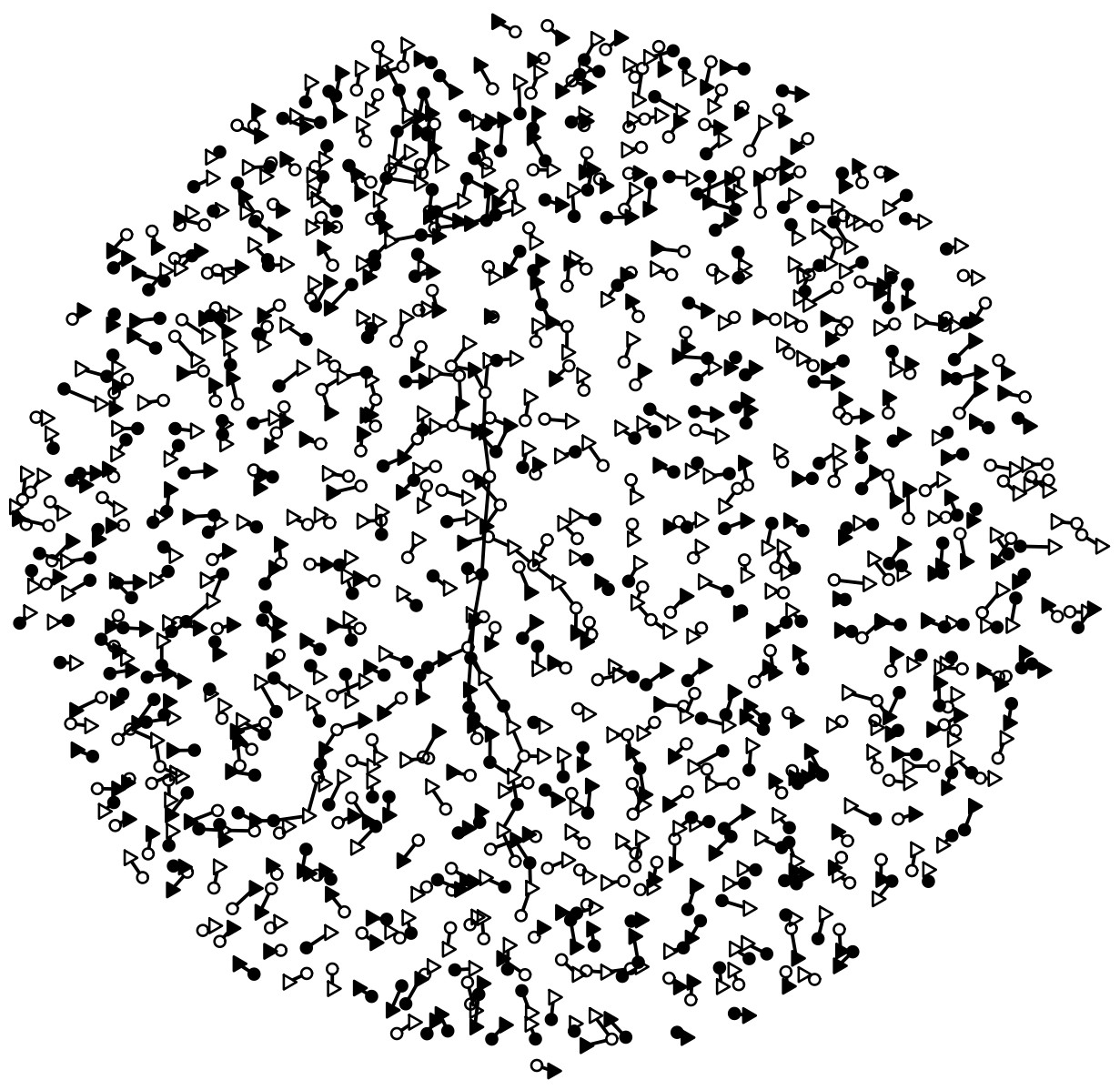

Fig S2: Network of partnerships between males (circles) and females (triangles) considered for Faux Add Health. The two colors represents different races. 
both sexes), symmetric degree distributions, and random (or proportional) mixing. We refer to this population as "Faux Symmetric." The degree distributions and cell-specific dependence parameters for Faux Symmetric are as shown in the top panel of Table S3, and degree and mixing totals for a population of 2,000 individuals are presented in the bottom panel. For Faux Symmetric, we constrained the network of partnerships to contain eight thousand 3-paths and five 4-cycles, and the plot of the network of partnerships (excluding isolates) is shown in Figure S3. This network is significantly more dense than Faux Add Health (2,000 partnerships as compared to 750 for Faux Add Health) due to both significantly lower numbers of isolates and higher prevalence of individuals of degrees two and higher. While unrealistic for most all sexual networks, such degree distributions and selective mixing totals may be reasonable for other types of networks, such as friendship or acquaintanceship networks.

TABLE S3

Degree distributions for non-Hispanic black and white males and females (top left) and cell-specific dependence parameters (top right), along with degree totals (bottom left) and mixing totals (bottom right) based on a population of 2,000 individuals for Faux

Symmetric.

\begin{tabular}{c|r} 
Degree & Proportion \\
\hline 0 & 0.20 \\
1 & 0.20 \\
2 & 0.20 \\
3 & 0.20 \\
4 & 0.20 \\
\hline
\end{tabular}

\begin{tabular}{cccc} 
& & \multicolumn{2}{c}{ Females } \\
& & \multicolumn{1}{c}{ Black } & White \\
\cline { 3 - 4 } Males & Black & $\alpha_{B B}=1$ & $\alpha_{B W}=1$ \\
& White & $\alpha_{W B}=1$ & $\alpha_{W W}=1$ \\
\cline { 3 - 4 } & & &
\end{tabular}

\begin{tabular}{c|rrrr} 
Degree & $\begin{array}{c}\text { Black } \\
\text { Males }\end{array}$ & $\begin{array}{r}\text { White } \\
\text { Males }\end{array}$ & $\begin{array}{c}\text { Black } \\
\text { Females }\end{array}$ & $\begin{array}{c}\text { White } \\
\text { Females }\end{array}$ \\
\hline 0 & 100 & 100 & 100 & 100 \\
1 & 100 & 100 & 100 & 100 \\
2 & 100 & 100 & 100 & 100 \\
3 & 100 & 100 & 100 & 100 \\
4 & 100 & 100 & 100 & 100 \\
\hline Pop. Size & 500 & 500 & 500 & 500 \\
\hline
\end{tabular}

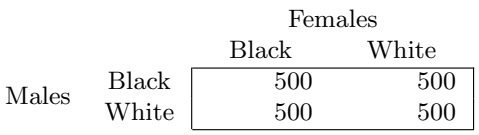

For both populations we consider simple random sampling of individuals (so no stratification by sex or race) with sample sizes constituting $25 \%$ of the total population size, producing a sample size of 500 individuals.

S6.2. Estimating Degree Distributions and Mixing Totals for a Given Population. To demonstrate that our method works well, we first consider simultaneous estimation of degree and mixing totals using constrained maximum likelihood estimation. Using 1,000 random samples of 500 individuals 


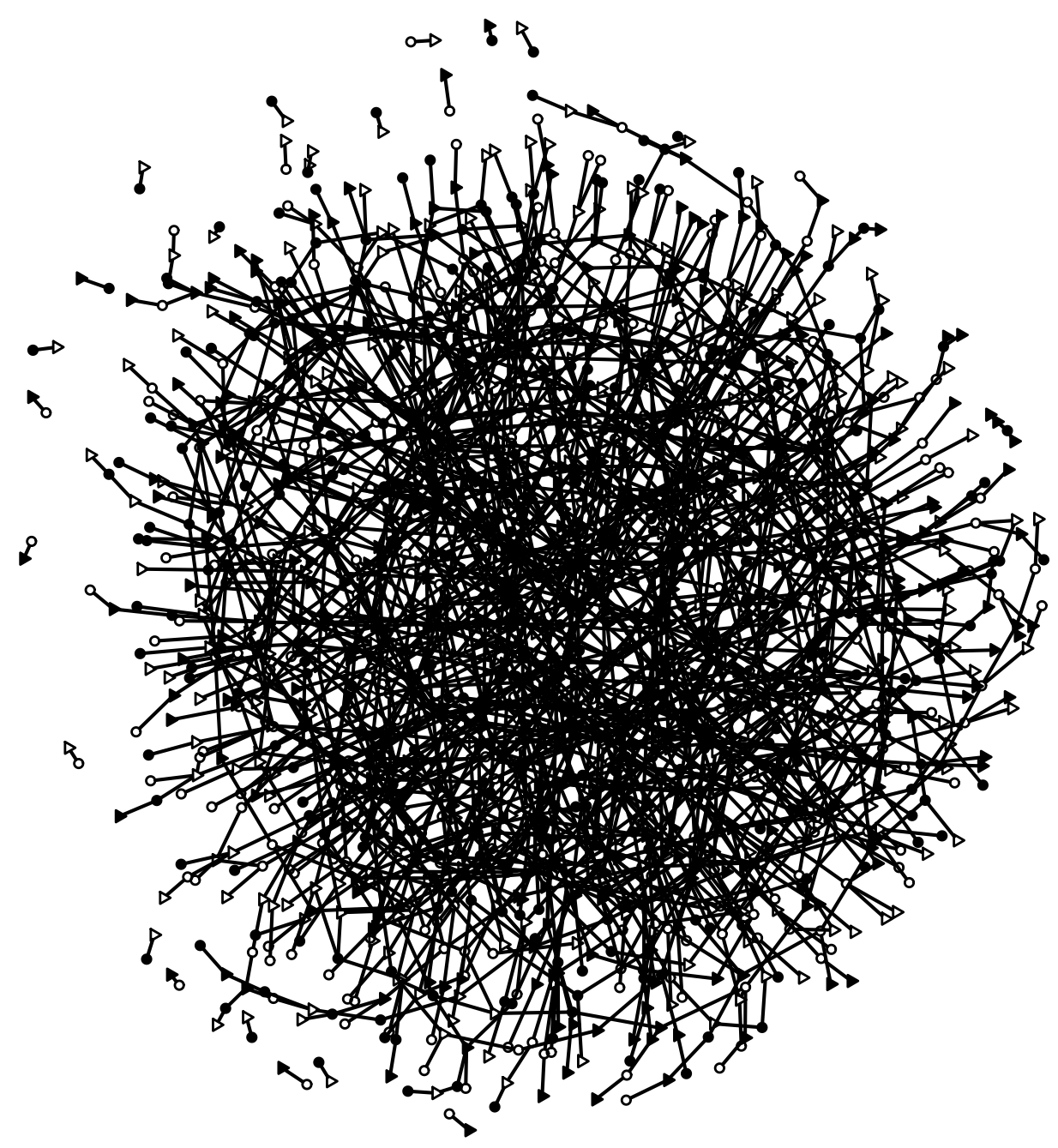

Fig S3: Network of partnerships between males (circles) and females (triangles) considered for Faux Symmetric. Isolates are excluded, and the two colors represents different races. 
from Faux Add Health and estimating population-level degree and mixing totals based on each sample using the method outlined in $\S 3.1$ of the paper, we obtain the distribution of degree totals presented in Figure S4. These show the simulated degree distributions for each sex and race being largely centered on the true population degree totals with only slight deviations for the number of non-Hispanic black males and females of degree 4 .

We note that these deviations are likely to be an artifact of the expected number of non-Hispanic black males (and females) of degree 4 in the sample being 0.5 , leading to an anticipated $50 \%$ of samples having no instances of such individuals. In such cases, the estimated degree distribution attributes a probability of 0 to that degree, meaning that the estimated population total corresponding to that degree is also 0 . This explains the median of 0 in the simulated distributions for degree 4 for both non-Hispanic black males and females.

If we consider similar simulations but where 1,500 individuals (i.e. $75 \%$ of the population) are sampled for each of the 1,000 simulations, these deviations disappear, and distributions of estimated degree totals are centered on the true population totals for all degrees, as demonstrated in the case of non-Hispanic black males and females in Figure S5. As would be anticipated under larger sample sizes, the variability in the distribution for each degree total is reduced from what is observed under sampling of $25 \%$ of the population. The estimated mixing totals are similarly effective in accurately targeting the true mixing totals, as demonstrated in Figure S6, where the true population totals again lie squarely in the center of simulated distributions.

Turning our attention to the Faux Symmetric population, if we carry out 1,000 separate simple random samples of $25 \%$ of the population, our method is again effective in producing estimated degree and mixing totals that accurately target the population totals, as demonstrated in Figure S7. As both races have equal representation in the population and the degree distribution is symmetric for the set of degrees considered, we would expect nearly identical distributions for simulated degrees for each race and sex. This is indeed what we appear to observe in Figure S7. Similarly, because this population has random mixing, the distribution of estimated selective mixing totals should be symmetric, and Figure S8 shows this to be the case. Thus, under both skewed and symmetric degree distributions and both assortative and random mixing, we find that our method is able to estimate degree distributions and selective mixing totals that are both consistent with each other and consistent with the underlying population. 

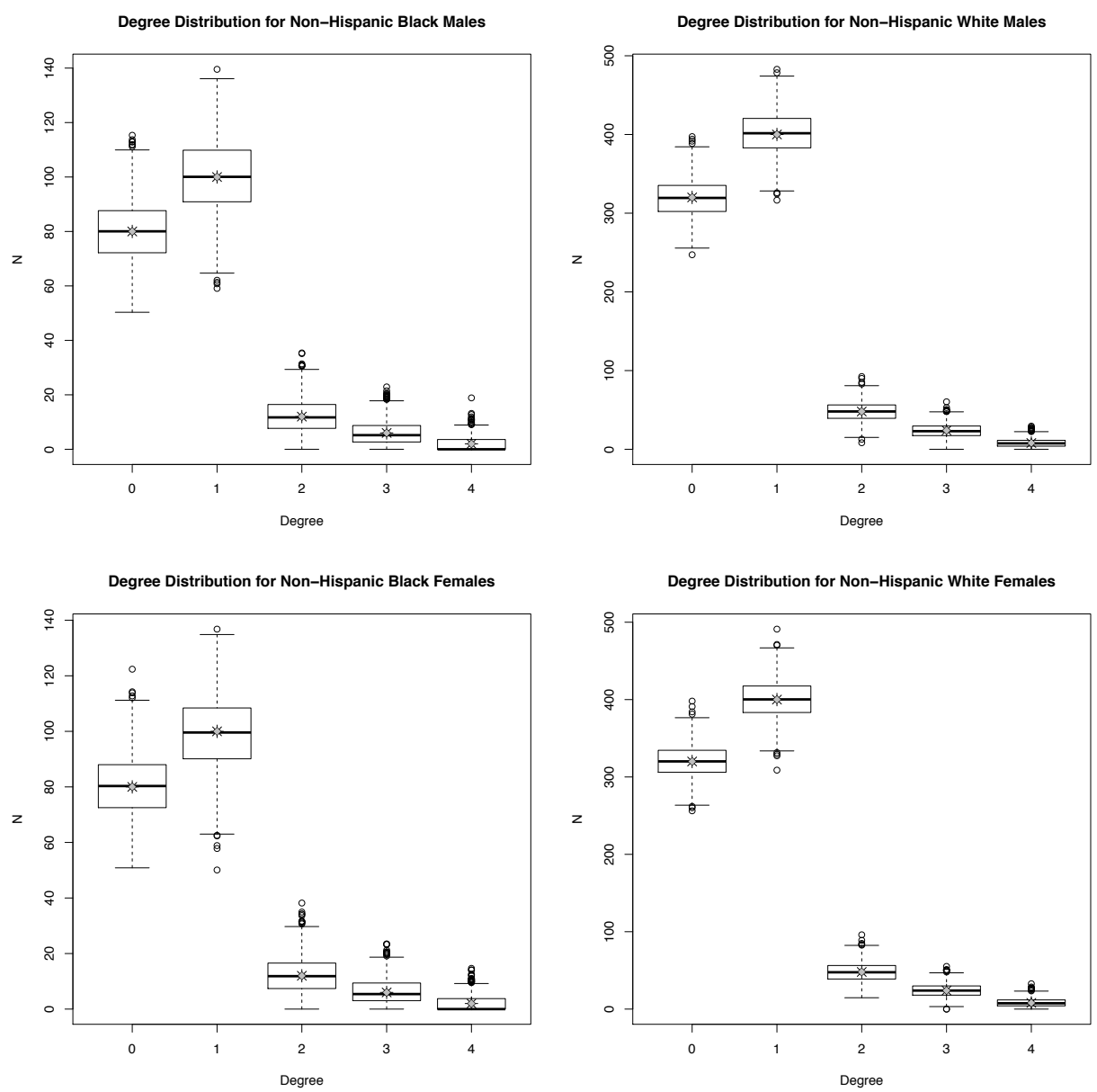

Fig S4: Estimated degree totals for non-Hispanic black males (top left), nonHispanic white males (top right), non-Hispanic black females (bottom left), and non-Hispanic white females (bottom right) for Faux Add Health under sampling of $25 \%$ of the population. Gray diamonds denote the population degree totals. 

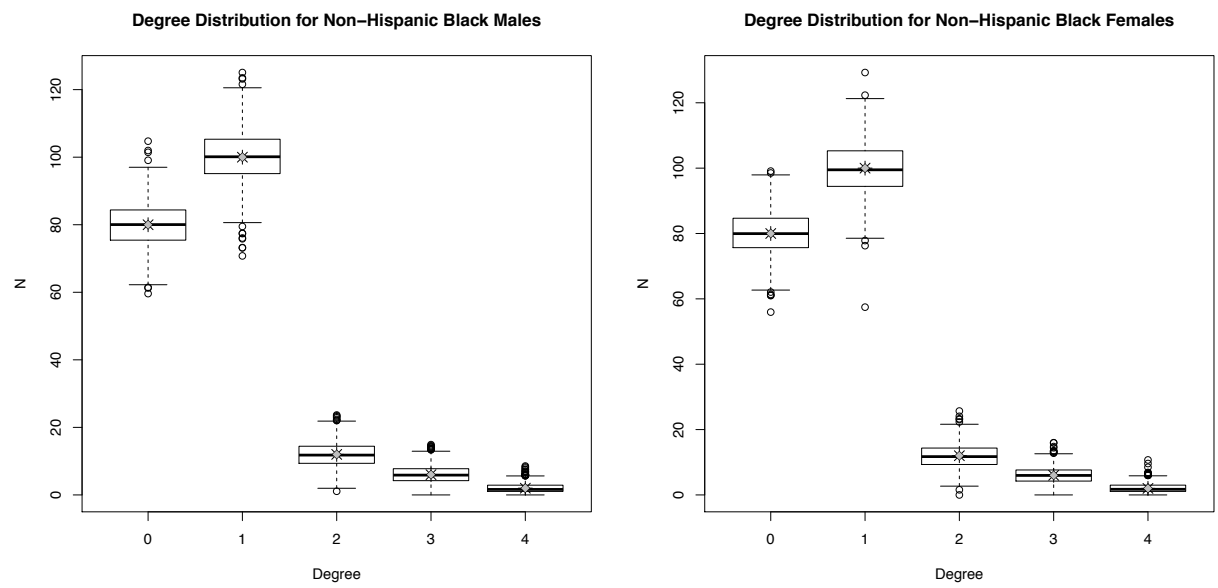

Fig S5: Estimated degree totals for non-Hispanic black males (left) and females (right) for Faux Add Health under sampling of $75 \%$ of the population. Gray diamonds denote the population degree totals.

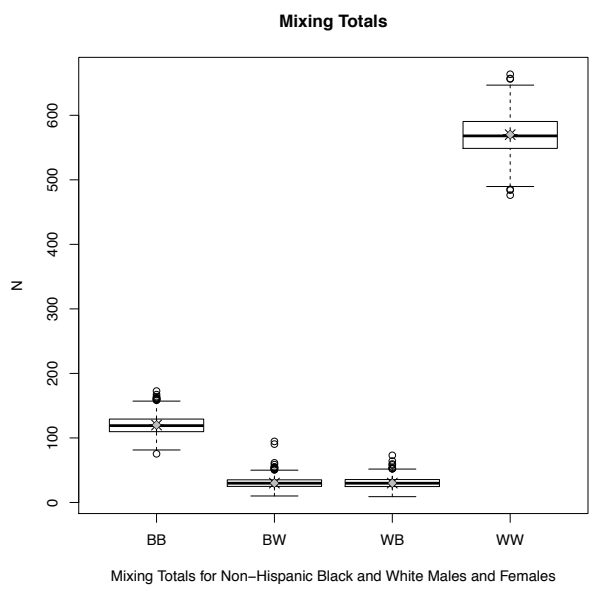

Fig S6: Estimated mixing totals for Faux Add Health under sampling of $25 \%$ of the population. Gray diamonds denote the population mixing totals. 

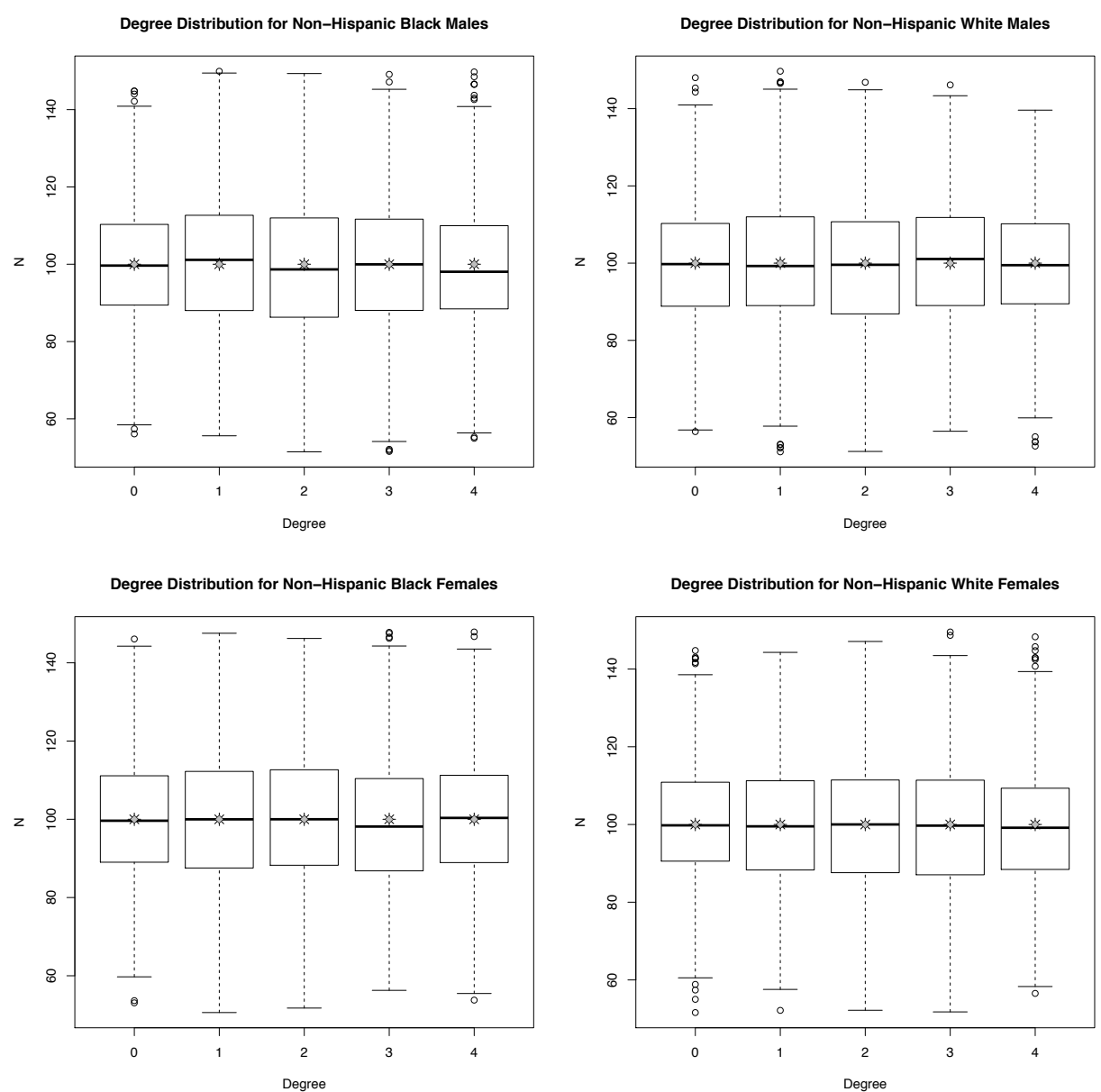

Fig S7: Estimated degree totals for non-Hispanic black males (top left), nonHispanic white males (top right), non-Hispanic black females (bottom left), and non-Hispanic white females (bottom right) for Faux Symmetric under sampling of $25 \%$ of the population. Gray diamonds denote the population degree totals. 


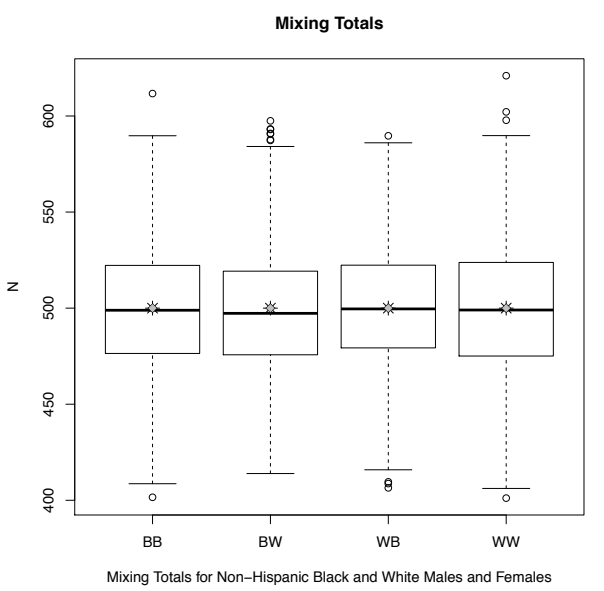

Fig S8: Estimated mixing totals for Faux Symmetric under sampling of 25\% of the population. Gray diamonds denote the population mixing totals.

S6.3. Generating Networks Consistent with Estimated Degree Distributions and Mixing Totals. Next, to show that the natural parameters of an ERGM estimated from mean value parameters can be used to generate random networks consistent with those specified mean value parameters, we estimate natural parameters for the ERGM given by

$$
\begin{aligned}
\log P\left(Y=y \mid \delta^{\mathcal{M}}, \delta^{\mathcal{F}}, \nu\right)= & \sum_{i=1}^{I} \sum_{k=1}^{F} \delta_{i k}^{\mathcal{M}} D_{i k}^{\mathcal{M}}(y)+\sum_{j=1}^{J} \sum_{\ell=1}^{M} \delta_{j \ell}^{\mathcal{F}} D_{j \ell}^{\mathcal{F}}(y) \\
& +\sum_{i=1}^{I} \sum_{j=1}^{J} \nu_{i j} N_{i j}(y)-\log \kappa\left(\delta^{\mathcal{M}}, \delta^{\mathcal{F}}, \nu\right)
\end{aligned}
$$

where $y$ is a realization of some random network $Y, D_{i k}^{\mathcal{M}}(y)$ is the number of males of type $i$ with momentary degree $k, D_{j \ell}^{\mathcal{F}}(y)$ is the number of females of type $j$ with momentary degree $\ell$, and $N_{i j}(y)$ is the number of partnerships between males of type $i$ and females of type $j$. The natural parameters $\delta^{\mathcal{M}}=\left\{\delta_{11}^{\mathcal{M}}, \ldots, \delta_{I F}^{\mathcal{M}}\right\}, \delta^{\mathcal{F}}=\left\{\delta_{11}^{\mathcal{F}}, \ldots, \delta_{J M}^{\mathcal{F}}\right\}$, and $\nu=\left\{\nu_{11}, \ldots, \nu_{I J}\right\}$ correspond to the sufficient statistics $D^{\mathcal{M}}=\left\{D_{11}^{\mathcal{M}}(y), \ldots, D_{I F}^{\mathcal{M}}(y)\right\}, D^{\mathcal{F}}=$ $\left\{D_{11}^{\mathcal{F}}(y), \ldots, D_{J M}^{\mathcal{F}}(y)\right\}$, and $N=\left\{N_{11}(y), \ldots, N_{I J}(y)\right\}$, and the expected value of these sufficient statistics is the vector of mean value parameters. Here, $\kappa\left(\delta^{\mathcal{M}}, \delta^{\mathcal{F}}, \nu, \zeta, \gamma\right)$ is the standard ERGM normalizing constant. We estimate the natural parameters using mean value parameters specified by the population degree distributions and mixing totals and applying the method 
outlined in $\S \mathrm{S} 5$ of the Supplement. For the estimated natural parameters and ERGM given by (S7), we simulate 1,000 networks using a burn-in of 100,000 iterations and interval length of 10,000. As explained in $\S 4$ of the paper, in estimating the natural parameters we truncate the degree distributions to ensure identifiability, so natural parameters are only estimated for degrees 0-3 (as opposed to 0-4) for each sex and race.

Using the population degree and mixing totals for Faux Add Health to estimate natural parameters for the ERGM given by (S7) and simulating 1,000 networks using this model, we obtain the distributions of simulated degrees by sex and race presented in Figure S9. These distributions are all centered on the mean value parameters. Similarly, simulated mixing totals accurately target the mean value parameters, as shown in Figure S10. We also present the distribution of 3-paths and 4-cycles for these simulated networks in Figure S10. Terms were not included in the ERGM for either 3-paths or 4-cycles, meaning that natural parameters were not estimated for either term, and it is readily apparent that the numbers of 3-paths and 4cycles from simulated networks do not target the population totals without bias.

As before, we consider similar simulations for Faux Symmetric. In this case, only the mean value parameters (and corresponding estimated natural parameters) change, so the ERGM is identical, and degree distributions are truncated in exactly the same manner as when considering Faux Add Health. As was the case for Faux Add Health, simulated networks produce degree and mixing totals consistent with mean value parameters, as evidenced by Figure S11 and Figure S12.

S6.4. Incorporating Additional Network Statistics. Finally, we consider an expansion of the ERGM specified by (S7) to include terms in addition to degree distribution and mixing totals. In particular, we focus on 3-paths and 4-cycles (both examples of dyad-dependent terms), although any of a number of network statistics could be considered here. The resulting ERGM considered has the form

$$
\begin{aligned}
\log P\left(Y=y \mid \delta^{\mathcal{M}}, \delta^{\mathcal{F}}, \nu, \zeta, \gamma\right)= & \sum_{i=1}^{I} \sum_{k=1}^{F} \delta_{i k}^{\mathcal{M}} D_{i k}^{\mathcal{M}}(y)+\sum_{j=1}^{J} \sum_{\ell=1}^{M} \delta_{j \ell}^{\mathcal{F}} D_{j \ell}^{\mathcal{F}}(y) \\
& +\sum_{i=1}^{I} \sum_{j=1}^{J} \nu_{i j} N_{i j}(y)+\zeta P+\gamma C \\
& -\log \kappa\left(\delta^{\mathcal{M}}, \delta^{\mathcal{F}}, \nu, \zeta, \gamma\right),
\end{aligned}
$$



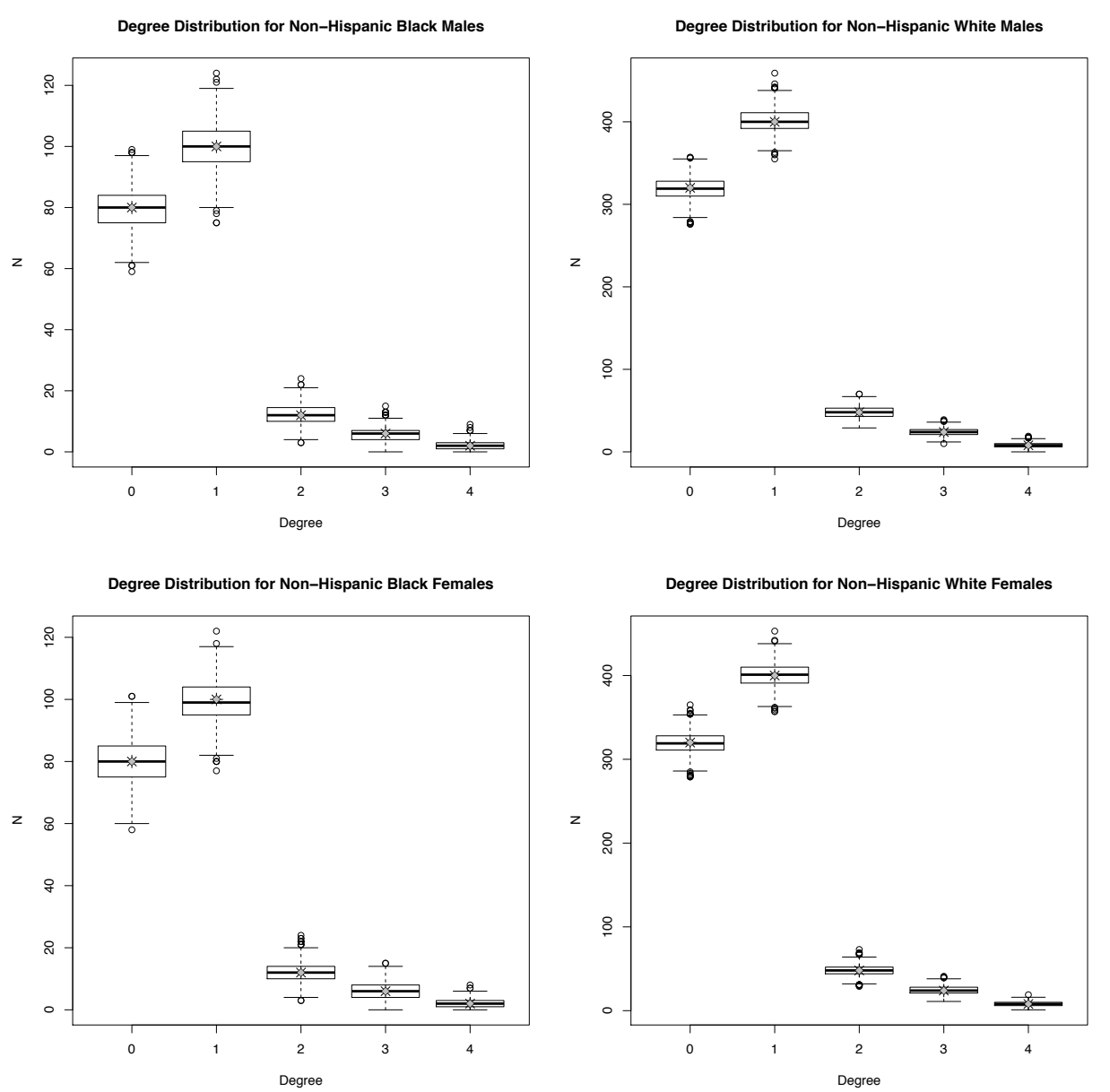

Fig S9: Simulated degree totals for non-Hispanic black males (top left), nonHispanic white males (top right), non-Hispanic black females (bottom left), and non-Hispanic white females (bottom right) for Faux Add Health. Gray diamonds denote the population degree totals. 

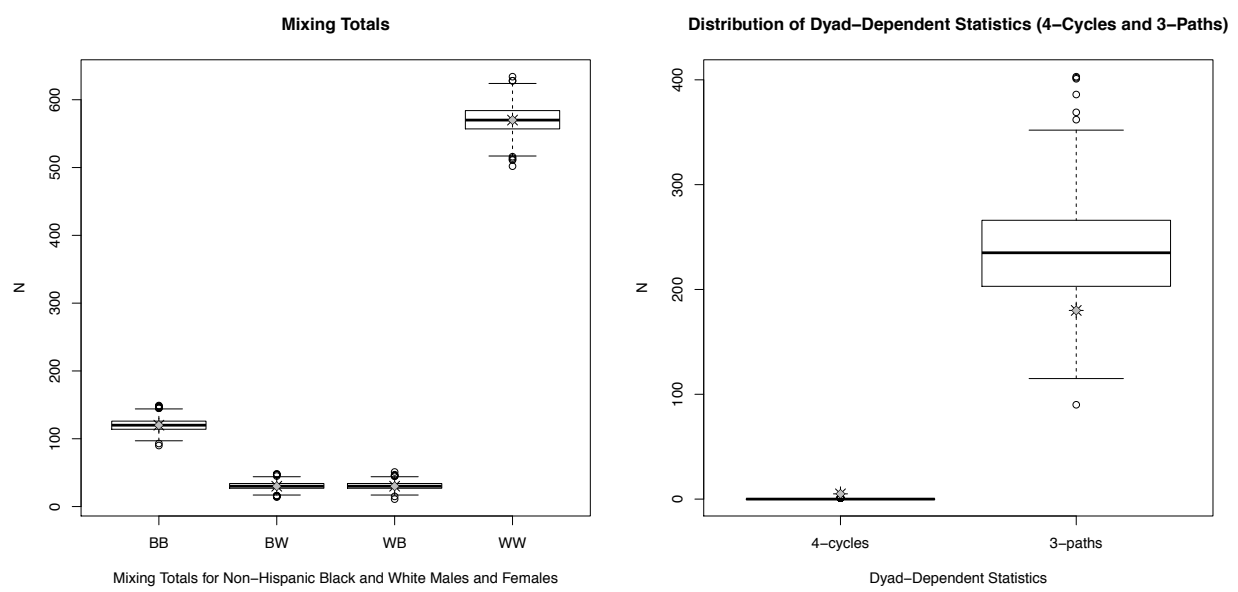

Fig S10: Simulated mixing totals (left) and 4-cycles and 3-paths (right) for Faux Add Health. Gray diamonds denote the population mixing totals, 4cycles, and 3-paths.

where parameters and sufficient statistics are as before except that additional natural parameters $\zeta$ and $\gamma$ are introduced corresponding to sufficient statistics $P$ and $C$, which represent the number of 3-paths and 4-cycles, respectively. If target mean value parameters are known (or else estimable from full or partial network data) for these additional model terms, then we can specify these in addition to population degree and mixing totals and apply the method of $\S S 5$ of the Supplement to estimate the natural parameters for this model.

If we recall the distributions of 3-paths and 4-cycles generated by simulated networks using the model given by (S7) and informed by degree and mixing totals for Faux Add Health, these produce networks with significantly more 3-paths and fewer 4-cycles than what are contained in the Faux Add Health Population. This is quite clear in Figure S10, where the true number of 4-cycles in the population (shown by the gray diamond) is at the extreme of the simulated distribution of 4-cycles, and the true number of 3 -paths lies in the lower quartile of the simulated distribution of 3-paths.

If we attempt to estimate natural parameters for (S8) using the population number of 3-paths (180) and 4-cycles (5) in addition to population degree and mixing totals as mean value parameters, the model is degenerate. This is because the targeted number of 4-cycles is extreme, as evidenced by Figure S10, so the vector of mean value parameters does not lie within the convex 

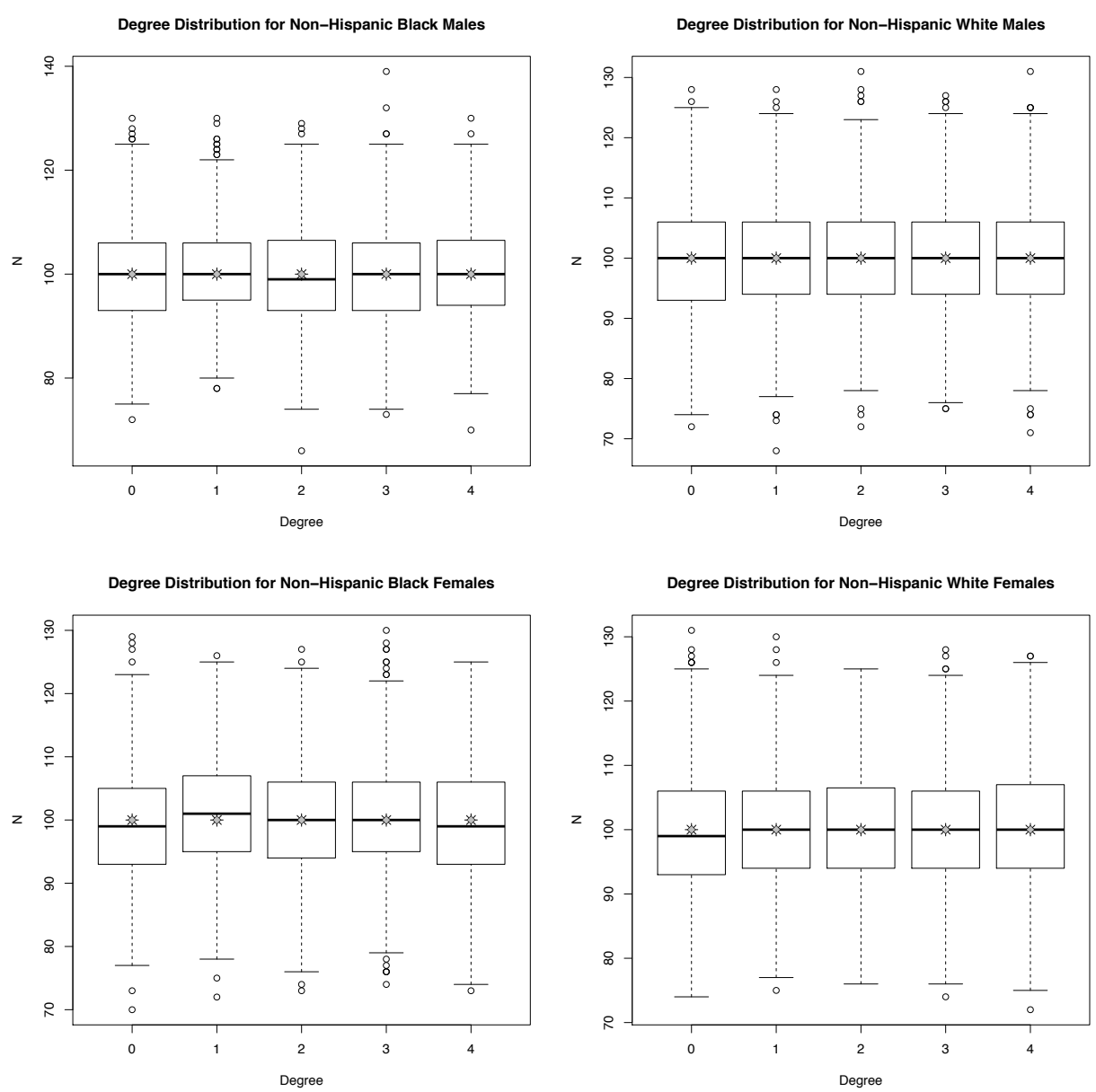

Fig S11: Simulated degree totals for non-Hispanic black males (top left), non-Hispanic white males (top right), non-Hispanic black females (bottom left), and non-Hispanic white females (bottom right) for Faux Symmetric. Gray diamonds denote the population degree totals. 


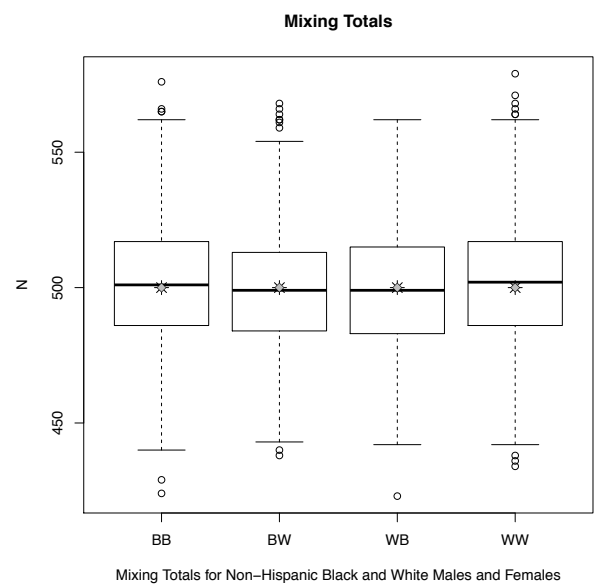

Fig S12: Simulated mixing totals for Faux Symmetric. Gray diamonds denote the population mixing totals.

hull of the sample space corresponding to the vector of sufficient statistics. If we remove the 4-cycles term but keep a 3-paths term in our model, we obtain a non-degenerate model, and 1,000 simulations based on this model produces the simulated distribution of 3-paths shown in Figure S13. These show that the model is now accurately targeting the desired number of 3-paths, so is able to at least produce networks consistent with the number of 3-paths in the population. (Simulated distributions for both degree and mixing totals are consistent with the target mean value parameters as before.)

For modelers who desire to incorporate a number of network statistics in their ERGM and use mean value parameters to estimate the corresponding natural parameters, the model degeneracy highlighted for 4-cycles for Faux Add Health is problematic, and it is important to understand when this is likely to occur. A simple diagnostic tool that can be implemented is to first consider a model consisting of degree and mixing totals and simulate networks based on this model. This can provide some sense of the sample space of the statistics corresponding to the other terms in the model, so one can examine the distributions for the other network statistics of interest and examine where target mean value parameters lie relative to those distributions. If, as we observed in the cause of 4-cycles for Faux Add Health, mean value parameters lie either on or very near the boundary of the convex hull of the sample space (i.e. at least one targeted mean value parameter lies at or near the extremes of its corresponding simulated distribution of statistics), then it can be anticipated that the model will be degenerate, and 


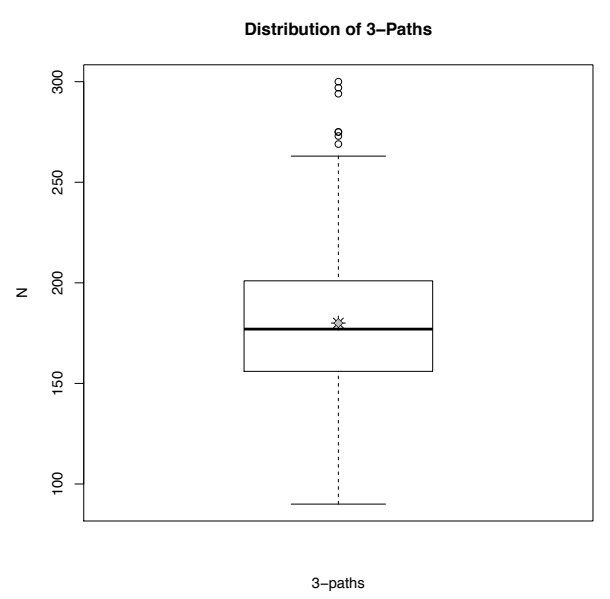

Fig S13: Simulated numbers of 3-paths for Faux Add Health for model incorporating degree totals, mixing totals, and a 3-path term. Gray diamonds denote the population number of 3-paths.

those network statistics for which target mean value parameters lie near the extremes of simulated distributions will either need to be removed or else have new targets for specified mean value parameters.

To demonstrate that the issue highlighted for 4-cycles for Faux Add Health is not a systemic issue, we again consider inclusion of terms for 3paths and 4-cycles for Faux Symmetric, using the ERGM given by (S8). Mean value parameters used include the population number of 3-paths $(8,000)$ and 4 -cycles $(5)$ in addition to degree and mixing totals. None of the targeted mean value parameters are extreme, and we are able to estimate the natural parameters for (S8) and simulate parameters consistent with the ERGM. In the case of 3-paths and 4-cycles, simulated distributions are as shown in Figure S14 and show that our method is again able to accurately target the desired mean value parameters. (Again, simulated distributions for both degree and mixing totals are consistent with the target mean value parameters.) Thus, our method is flexible and allows modelers to target a variety of network statistics simultaneously, meaning that modelers can generate initial networks that are consistent not only with dyad-independent statistics like momentary degree distributions and selective mixing totals but also dyad-dependent statistics such as number of 3-paths and 4-cycles.

\section{References.}

L. Abu-Raddad, P. Patnaik, and J.G. Kublin. Dual infection with HIV and malaria fuels the spread of both diseases in sub-Saharan Africa. Science, 314:1603-1606, 2006.

imsart-aoas ver. 2012/08/31 file: Supplement2.tex date: June 4, 2016 

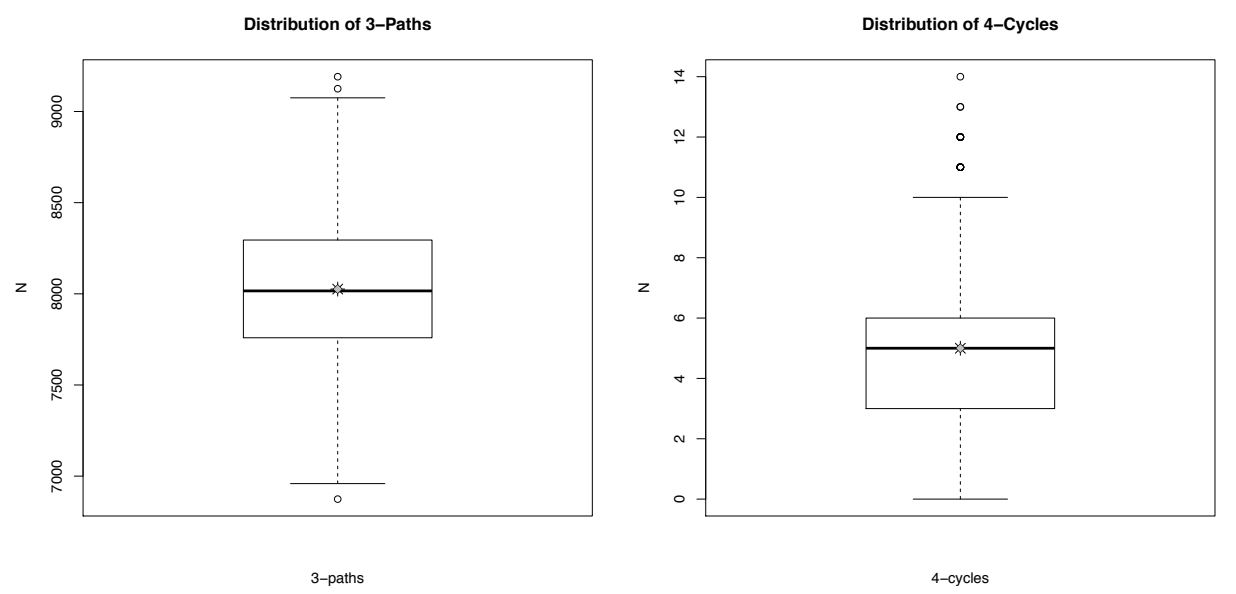

Fig S14: Simulated numbers of 3-paths (left) and 4-cycles (right) for Faux Symmetric. Gray diamonds denote the population numbers of 3-paths and 4-cycles.

A.A. Adimora and V.J. Schoenbach. Contextual factors and the black-white disparity in heterosexual HIV transmission. Epidemiology, 13(6):707-712, 2002.

A.A. Adimora and V.J. Schoenbach. Social context, sexual networks, and racial disparities in rates of sexually transmitted infections. Journal of Infectious Diseases, 191 (Supplement):S115-S122, 2005.

A.A. Adimora, V.J. Schoenbach, and I.A. Doherty. HIV and African Americans in the southern United States: Sexual networks and social context. Sexually Transmitted Diseases, 33 (Supplement)(7):S39-S45, 2006.

A.A. Adimora, V.J. Schoenbach, and I.A. Doherty. Concurrent sexual partnerships among men in the United States. American Journal of Public Health, 97(12):2230-2237, 2007.

O.E. Barndorff-Nielsen. Information and Exponential Families in Statistical Theory. Wiley, New York, 1978.

N.B. Carnegie and M. Morris. Size matters: Concurrency and the epidemic potential of HIV in small networks. PLoS One, 7:e43048, 2011.

S.E. Chick, A.L. Adams, and J.S. Koopman. Analysis and simulation of a stochastic, discrete-individual model of STD transmission with partnership concurrency. Mathematical Biosciences, 166(1):45-68, 2000.

K.R. Davis and S.C. Weller. The effectiveness of condoms in reducing heterosexual transmission of HIV. Family Planning Perspectives, 31(6):272-279, 1999.

Demographic and Health Surveys Program. HIV/AIDS Survey Indicators Database. http: //hivdata.dhsprogram.com, 2015. Accessed: December 1, 2015.

I.A. Doherty, S. Shiboski, J.M. Ellen, A.A. Adimora, and N.S. Padian. Sexual bridging socially and over time: a simulation model exploring the relative effects of mixing and concurrency on viral sexually transmitted infection transmission. Sexually Transmitted Diseases, 33(6):368-373, 2006.

J.W. Eaton, T.B. Hallett, and G.P. Garnett. Concurrent sexual partnerships and primary 
HIV infection: A critical interaction. AIDS and Behavior, 15(4):687-692, 2011.

J.W. Eaton, N. McGrath, and M.-L. Newell. Unpacking the recommended indicator for concurrent sexual partnerships. AIDS, 26(8):1037-1039, 2012.

D.T. Fleming and J.N. Wasserheit. From epidemiological synergy to public health policy and practice: The contribution of other sexually transmitted diseases to sexual transmission of HIV infection. Sexually Transmitted Infections, 75(1):3-17, 1999.

O. Frank and D. Strauss. Markov graphs. Journal of the American Statistical Association, 81(395):832-842, 1986.

L. Gaydosh, G. Reniers, and S. Helleringer. Partnership concurrency and coital frequency. AIDS and Behavior, 17(7):2376-2386, 2013.

C.J. Geyer and E.A. Thompson. Constrained Monte Carlo maximum likelihood for dependent data. Journal of the Royal Statistical Society, Series B, 54(3):657-699, 1992.

A.C. Ghani and G.P. Garnett. Risks of acquiring and transmitting sexually transmitted diseases in sexual partner networks. Sexually Transmitted Diseases, 27(10):579-587, 2000 .

A.C. Ghani, J. Swinton, and G.P. Garnett. The role of sexual partnership networks in the epidemiology of gonorrhea. Sexually Transmitted Diseases, 24:45-56, 1997.

S.M. Goodreau. A decade of modelling research yields considerable evidence for the importance of concurrency: A response to Sawers and Stillwaggon. Journal of the International AIDS Society, 14(12):1-7, 2011.

S.M. Goodreau, S. Cassels, D. Kasprzyk, D.E. Montaño, A. Greek, and M. Morris. Concurrent partnerships, acute infection and HIV epidemic dynamics among young adults in Zimbabwe. AIDS and Behavior, 6(2):312-322, 2012.

D.T. Hamilton and M. Morris. The racial disparities in STI in the U.S.: Concurrency, STI prevalence, and heterogeneity in partner selection. Epidemics, 11:56-61, 2015.

D.T. Hamilton, M.S. Handcock, and M. Morris. Degree distributions in sexual networks: A framework for evaluating evidence. Sexually Transmitted Diseases, 35:30-40, 2008.

M.S. Handcock. Assessing degeneracy in statistical models of social networks. Working paper, Center for Statistics and the Social Sciences, University of Washington, 2003.

K.M. Harris, C.T. Halpern, E. Whitsel, J. Hussey, J. Tabor, P. Entzel, and J.R. Udry. The National Longitudinal Study of Adolescent Health: Research Design [www document]. http://www.cpc.unc.edu/projects/addhealth/design. Technical report, Carolina Population Center, University of North Carolina at Chapel Hill, Available at: http://www.cpc.unc.edu/projects/addhealth/design, 2009.

S. Helleringer, J. Mkandawire, and H.-P. Kohler. A new approach to measuring partnership concurrency and its association with HIV risk in couples. AIDS and Behavior, 18:22912301, 2014.

P.W. Holland and S. Leinhardt. An exponential family of probability distributions for directed graphs. with comments by Ronald L. Breiger, Stephen E. Fienberg, Stanley S. Wasserman, Ove Frank and Shelby J. Haberman and a reply by the authors. Journal of the American Statistical Association, 76(373):33-65, 1981. ISSN 0162-1459.

T.D. Hollingsworth, R. Anderson, and C. Fraser. HIV-1 transmission, by stage of infection. Journal of Infectious Diseases, 198:687-693, 2008.

C. Hudson. Concurrent partnerships could cause AIDS epidemics. International Journal of STD and AIDS, 4:349-353, 1993.

D.R. Hunter and M.S. Handcock. Inference in curved exponential family models for networks. Journal of Computational and Graphical Statistics, 15(3):565-583, 2006.

D.R. Hunter, M.S. Handcock, C.T. Butts, S.M. Goodreau, and M. Morris. ergm: A package to fit, simulate and diagnose exponential-family models for networks. Journal of Statistical Software, 24(3), 2008.

imsart-aoas ver. 2012/08/31 file: Supplement2.tex date: June 4, 2016 
L.F. Johnson, R.E. Dorrington, D. Bradshaw, V. Pillay-Van Wyk, and T.M. Rehle. Sexual behaviour patterns in South Africa and their association with the spread of HIV: Insights from a mathematical model. Demographic Research, 21(11):289-339, 2009.

E.F. Kjetland, P.D. Leutscher, and P.D. Ndhlovu. A review of female genital schistosomiasis. Trends Parasitology, 28:58-65, 2012.

J.S. Koopman, S.E. Chick, C.S. Riolo, A.L. Adams, M.L. Wilson, and M.P. Becker. Modeling contact networks and infection transmission in geographic and social space using GERMS. Sexually Transmitted Diseases, 27(10):617-626, 2000.

M. Kretzschmar and M. Caraël. Is concurrency driving HIV transmission in Sub-Saharan African sexual networks? The significance of sexual partnership typology. AIDS and Behavior, 16:1746-1752, 2012.

M. Kretzschmar and M. Morris. Measures of concurrency in networks and the spread of infectious disease. Mathematical Biosciences, 133:165-195, 1996.

E. Lagarde, B. Auvert, Caraël, M. Laorou, B. Ferry, E. Akam, T. Sukwa, L. Morison, B. Maury, J. Chege, I. N'Doye, A. Buvé, and Study Group on Heterogeneity of HIV Epidemics in African Cities. Concurrent sexual partnerships and HIV prevalence in five urban communities of Sub-Saharan Africa. AIDS, 15(7):877-884, 2001.

M.N. Lurie and S. Rosenthal. Concurrent partnerships as a driver of the HIV epidemic in Sub-Saharan Africa? The evidence is limited. AIDS and Behavior, 14:17-24, 2010.

T.L. Mah and D.T. Halperin. Concurrent sexual partnerships and the HIV epidemics in Africa: Evidence to move forward. AIDS and Behavior, 14:11-16, 2010.

V. Mishra and S.B.-V. Assche. Concurrent Sexual Partnerships and HIV infection: Evidence from National Population-Based Surveys. DHS Working Papers No. 62, Macro International Inc., Calverton, 2009.

M. Morris and M. Kretzschmar. Concurrent partnerships and transmission dynamics in networks. Social Networks, 17:299-318, 1995.

M. Morris and M. Kretzschmar. Concurrent partnerships and the spread of HIV. AIDS, 5(11):641-648, 1997.

M. Morris and M. Kretzschmar. A microsimulation study of the effect of concurrent partnerships on the spread of HIV in Uganda. Mathematical Population Studies, 8(2): 109-133, 2000.

M. Morris, S.M. Goodreau, and J. Moody. Sexual networks, concurrency, and STD/HIV. In K.K. Holmes, P.F. Sparling, W.E. Stamm, P. Piot, J.N. Wasserheit, L. Corey, and D.H. Watts, editors, Sexually Transmitted Diseases, pages 109-125. McGraw-Hill, New York, $4^{\text {th }}$ edition, 2007.

M. Morris, M.S. Handcock, and D.R. Hunter. Specification of exponential-family random graph models: Terms and computational aspects. Journal of Statistical Software, 24(4), 2008.

M. Morris, A.E. Kurth, D.T. Hamilton, J. Moody, and S. Wakefield. Concurrent partnerships and HIV prevalence disparities by race: Linking science and public health practice. American Journal of Public Health, 99(6):1023-1031, 2009.

NIMH Collaborative HIV/STD Prevention Trial Group. Methodological overview of a fivecountry community-level $\mathrm{HIV} /$ sexually transmitted disease prevention trial. AIDS, 21 (2 (Supplement)):S3-S18, 2007.

I.F. Sandøy, K. Dzekedzeke, and K. Fylkesnes. Prevalence and correlates of concurrent sexual partnerships in Zambia. AIDS and Behavior, 14(1):59-71, 2010.

L. Sawers. Measuring and modelling concurrency. Journal of the International AIDS Society, 16(1):1-20, 2013.

L. Sawers and E. Stillwagon. Concurrent sexual partnerships do not explain the HIV epidemics in Africa: a systematic review of the evidence. Journal of the International

imsart-aoas ver. 2012/08/31 file: Supplement2.tex date: June 4, 2016 
AIDS Society, 13(34):1-23, 2010.

D. Strauss and M. Ikeda. Pseudolikelihood estimation for social networks. Journal of the American Statistical Association, 85(409):204-212, 1990.

R. Szabo and R.V. Short. How does male circumcision protect against HIV infection? British Medical Journal, 320(7249):15921594, 2010.

F. Tanser, T. Bärnighausen, L. Hund, G. Garnett, N. McGrath, and M.-L. Newell. Effect of concurrent sexual partnerships on rate of new HIV infections in a high prevalence, rural South African population: a cohort study. Lancet, 378(9787):247-255, 2011.

UNAIDS Reference Group on Estimates, Modelling, and Projections. Consultation on Concurrent Sexual Partnerships: Recommendations from a meeting of the UNAIDS Reference Group on Estimates, Modelling and Projections held in Nairobi, Kenya, April 20-21 $1^{\text {st }}$ 2009. UNAIDS, Nairobi, 2009.

S. Wasserman and P. Pattison. Logit models and logistic regression for social networks: I. An introduction to Markov graphs and $p^{*}$. Psychometrika, 61(3):401-425, 1996.

C. H. Watts and Robert M. May. The influence of concurrent partnerships on the dynamics of HIV/AIDS. Mathematical Biosciences, 108:89-104, 1992.

K. Wellings, M. Collumbien, E. Slaymaker, S. Singh, Z. Hodges, D. Patel, and N. Bajos. Sexual behavior in context: A global perspective. Lancet, 368(9548):1706-1728, 2006. 